
\title{
25 Research Square \\ Urine phthalate levels and liver function in US adolescents: Analyses of NHANES 2007-2016
}

\section{Shiting Xiang ( 2644900547@qq.com )}

Hunan Children's Hospital https://orcid.org/0000-0002-8191-6541

Jie Dong

Hunan Children's Hospital

Xun Li

Hunan Children's Hospital https://orcid.org/0000-0002-5070-8950

Liping Li

Hunan Children's Hospital

Chao Li

Central South University

\section{Research Article}

Keywords: Phthalate, Liver, Adolescents

Posted Date: November 5th, 2021

DOI: https://doi.org/10.21203/rs.3.rs-987618/v1

License: (c) (1) This work is licensed under a Creative Commons Attribution 4.0 International License. Read Full License

Version of Record: A version of this preprint was published at Frontiers in Public Health on March 4th, 2022. See the published version at https://doi.org/10.3389/fpubh.2022.843971. 


\section{Abstract}

Phthalates are non-persistent chemicals with endocrine-disrupting abilities widely used in a variety of consumer products. Evidence for the effects of phthalate exposure on liver function in adolescents is lacking. The aim of this study was to investigate the association between urine phthalate levels and changes in liver function indicators. Methods: Data were analyzed from the combined 2007-2016 National Health and Nutrition Examination Survey (NHANES). Weighted linear regressions were used to explore the association between urinary phthalate metabolites and indicators of liver function. Bayesian kernel machine regressions (BKMR) were used to evaluate the joint effects of phthalate metabolite mixtures on indicators of liver function. Results: Weighted Linear regression models showed that MCOP and MiBP were negatively associated with TBIL (all P FDR < 0.05), MCPP was positively correlated with TBIL (P FDR < 0.05), $\Sigma D E H P, ~ M C O P$, and MEP (all P FDR < 0.05) were negatively correlated with ALB, while MCPP was positively correlated with ALB (P FDR < 0.05), and MCOP was negatively correlated with TP ( FDR < 0.05). BKMR analysis showed phthalate metabolite mixtures had significant positive dose response relationships with $A L T, A S T, G G T, A L P$ and TBIL, and significant negative dose response relationships with ALB and TP. Conclusions: Phathalate metabolites were associated with changes in liver function indicators among U.S. adolescents.

\section{Introduction}

Phthalates, known as plasticizers, are non-persistent chemicals with endocrine-disrupting abilities widely used in a variety of consumer products (Katsikantami et al. 2016). High molecular weight phthalates, including di-(2-ethylhexyl) phthalate (DEHP) and di-isononyl phthalate (DiNP), are used primarily as plasticizer for polyvinyl chloride, building and construction materials, and several categories of toys (such as plastic books, ball, doll and cartoon characters). Low molecular weight phthalates, including di-butyl phthalate (DBP) and diethyl phthalate (DEP), are used primarily as fragrance ingredients in cosmetics, home and personal care products (Odebeatu et al. 2019; Wang et al. 2018). As phthalates are usually bound to polymers by non-chemical bonds, they are often constantly released from plastic products into the surrounding environment, resulting in food, water or air pollution (Benjamin et al. 2017). Human are exposed to large amounts of phthalates through dietary, inhalation and skin contact (Benjamin et al. 2017).

Liver plays an important role in the detoxification of phthalates (Praveena et al. 2018). The hepatotoxicity of phthalates has been demonstrated in animal models such as mice, zebrafish, and quail (Ito et al.2019; Zhang et al.2019; Jiao et al. 2019). Phthalate concentrations have been adversely associated with indicators of liver function in adulthood [9], but few studies have examined associations between phthalate exposure and liver function in youth. Liver disease is a major cause of illness and death worldwide. Approximately 2 million people die from it every year in the world (Asrani et al. 2019). While vaccination and newer drugs will reduce the burden of viral-related liver disease (Asrani et al. 2019), liver disorders are highly prevalent in general population adolescents (Lawlor et al. 2014). The need for liver disease research is even more obvious. 
Therefore, in the present study, we aimed to examine the association between phthalate exposure and indicators of liver function using a nationally representative sample of adolescents aged 12-19 years in the United States. We hypothesized that urine phthalate concentrations would have predictive significances for altered liver function indicators in US adolescents.

\section{Materials And Methods \\ 2.1 Study population}

National Health and Nutrition Examination Survey (NHANES) is a cross-sectional, nationally representative survey in the United States conducted annually by CDC's National Center for Health Statistics (CDC/NCHS). A detailed description of the study design can be found elsewhere (CDC 2016). The survey uses a multistage stratified probability sample based on selected counties, blocks, households, and persons within households. Survey interviews were conducted in participants' homes by well-trained professionals, while extensive physical examinations, including blood and urine collection, were conducted at mobile exam centers.

The present analysis included five waves of the NHANES from 2007 to 2016, which were publicly shared and downloaded from the CDC official website and combined according to the NHANES tutorials. There were 50588 subjects from these years, of which 6598 were aged between 12 and 19 years. First, we excluded subjects who were serologically positive for hepatitis $B$ virus or hepatitis $C$ virus. We then excluded samples from the analysis that did not have complete records including liver function test and phthalate. Further, missing data samples for covariables such as poverty income ratio, body mass index and physical activity were excluded. Finally, 6 samples with total bilirubin (TBIL) test value of 0 were deleted, and a total of 1650 adolescents were selected as final samples. (Supplementary Figure 1).

\subsection{Liver function measure outcomes}

Fasting blood samples were collected in NHANES participants aged 12 years and older at a mobile examination center. The samples were refrigerated and transported to the central laboratory for analysis of serum liver function indicators using the Beckman Coulter DxC800 Synchron clinical system (Zipf et al. 2013).

The liver is rich in alanine aminotransferase (ALT) and aspartate aminotransferase (AST). Serum levels of these two enzymes rise when hepatocytes necrosis or liver cell membrane damage (Whitehead et al.1999). AST/ALT ratio is used for differential diagnosis of acute and chronic liver diseases. The liver is the only place where albumin (ALB) is synthesized. When liver function is impaired, serum albumin (ALB) and total protein (TP) levels decrease (Whitfield et al 2001). Alkaline phosphotase (ALP) and Gamma glutamyl transferase (GGT) are markers of cholestasis (Gazzin et al. 2016). The liver has the functions of uptake, combination and excretion of bilirubin metabolism. The disorder of one or more functions can lead to the increase of total bilirubin (TBIL) (Newsome et al. 2018).

\subsection{Measurement of phthalate}


Phthalate metabolites were measured in spot urine samples from a third of study subjects randomly selected from participants six years of age and older. The collected samples were frozen at $-20{ }^{\circ} \mathrm{C}$ and then shipped to the CDC's National Center for Environmental Health for analysis. Urine specimens were processed using high performance liquid chromatography-electrospray ionization-tandem mass spectrometry (HPLC-ESI-MS/MS) for the quantitative detection of phthalate metabolites (Zipf et al. 2013).

We selected 12 metabolites tested in all 5 rounds and excluded phthalate metabolites whose measured values were more than $40 \%$ below the detection limit (LOD). The remaining 11 urinary phthalate metabolites used in our study were mono-(carboxyisononyl) phthalate (MCNP), mono-(carboxyisoctyl) phthalate (MCOP), mono-2-ethyl-5-carboxypentyl phthalate (MECPP), mono-n-butyl phthalate (MnBP), mono-(3-carboxypropyl) phthalate (MCPP), mono-ethyl phthalate (MEP), mono-(2-ethyl-5-hydroxyhexyl) phthalate (MEHHP), mono-(2-ethylhexyl phthalate (MEHP), mono-isobutyl phthalate (MiBP), mono-(2ethyl-5-oxohexyl) phthalate (MEOHP), and mono-benzyl phthalate (MBzP). Phthalate metabolites concentrations below LODs were replaced with LOD divided by the square root of two.

Concentrations of MECPP, MEHHP, MEHP and MEOHP were divided by their respective molar weight (MW) to obtain the molar equivalent. We summed the molar equivalents of these metabolites and multiplied by the molar weight of MEHP (MW=278) to obtain LDEHP metabolites (Wolff et al.2010).

\subsection{Measurements of Covariates}

Covariates were selected as potential confounders by referencing to previous publications (Attanasio et al. 2019; Malin et al 2019). Sociodemographic covariates included gender (Male, Female), race (NonHispanic White, Non-Hispanic Black, Mexican American, Other Hispanic, Other/Mixed), education (Less than high school, High School graduate or GED, More than High), and Ratio of family income to poverty $(\leq 1,>1)$. Lifestyle covariate included physical activity. It was measured by respondents' self-reported time spent in vigorous or moderate recreational physical activity. The dichotomous variables for physical activity were classified as "inactive" ( $<10$ min per week) and "activity" ( $\geq 10$ min per week). Examination results covariate included body mass index (BMI). BMI was calculated as weight $(\mathrm{kg}) /$ height2 (m2) measured in the physical examination and categorized into three levels: $<25 \mathrm{~kg} / \mathrm{m} 2$ (Normal/Underweight), $25<30 \mathrm{~kg} / \mathrm{m} 2$ (overweight) and $\geq 30 \mathrm{~kg} / \mathrm{m} 2$ (obese) (Frediani et al. 2018).

\subsection{Statistical analysis}

Demographic characteristics were reported as percentages. Phthalate metabolite concentrations and liver function levels were described in the quartile range. We used urine creatinine to adjust the concentrations of phthalate metabolites in all statistical analyses. Creatinine-adjusted phthalate metabolites concentrations and indicators of liver function were natural log-transformed to make them normally distributed. Spearman's coefficients were used to test the pairwise correlations of phthalate metabolite concentrations (Supplementary Table 2). We performed survey-weighted linear regression to assess the associations of the urinary phthalate metabolites with indicators of liver function. Benjamini-Hochberg false discovery rate (FDR) correction was used to adjust $\mathrm{P}$ values to adjust for multiple testing. 
We further performed a BKMR analysis to evaluate the joint effects of exposure to phthalate metabolite mixtures on indicators of liver function (Bobb et al. 2015). We fitted separate BKMR models for each index of liver function according to the following models:

$\mathrm{Yi}=\mathrm{h}($ (LDEHPi, MCNPi, MCOPi, MnBPi, MCPPi, MEPi, MiBPi, MBzPi) $+\beta z i+e i$. The $\mathrm{h}()$ is an exposureresponse function, which can incorporate non-linear relationships and interactions among the mixture components; $\beta$ is coefficient; $z i$ is covariates. BKMR identifies the relative importance of individual exposure variables to the joint effects by providing an estimate of posterior inclusion probabilities (PIPs). The PIP threshold was 0.5 in this study (Shah-Kulkarni et al.2020). BKMR facilitates visualization of results for the effect of single exposure and interactions between exposure and outcome. First, the cumulative effect of exposure to the combination of eight phthalate metabolites on indicators of live function in adolescents was evaluated. Then, the effects of a single exposure to a single phthalate metabolite were calculated when other phthalate metabolites were fixed to their median level. Lastly, the joint effect of two phthalate metabolites were studied by plotting a dose-response relationship of one phthalate metabolite at different quantiles of another phthalate metabolite, based on the median level of the other phthalate metabolites.

All models were adjusted for ratio of family income to poverty, BMI, age, gender, race, education, and physical activity. All analyses were performed using phthalate-specific subsample weight as recommended by NCHS, to account for the complex sampling design and non-response of NHANES. Weights for combined NHANES survey cycles were calculated according to NHANES guidelines. All statistical analyses were performed using $R$ 3.5.3. All test values were 2-sided and $P<0.05$ was considered significant.

\section{Results}

\subsection{Study population}

Characteristics of the study subjects are shown in Table 1. Of the 1650 participants, the average age was $15.49 \pm 2.266$ years, with female subjects accounting for $46.5 \%$. Most of the participants are Non-Hispanic White, $84.4 \%$ of the participants had education less than high school, $67.3 \%$ had a ratio of family income to poverty greater than $1,16.1 \%$ were obese, and $77.9 \%$ were physically active (Table 1 ). 
Table 1

Demographic characteristics for adolescents aged

12-19 years old in NHANES 2007-2016 $(\mathrm{N}=1,650)$

\begin{tabular}{|lcc|}
\hline Basic characteristics & N & $\%$ \\
\hline Age(years) & & \\
\hline $12-14$ & 631 & 38.2 \\
\hline $15-17$ & 618 & 37.5 \\
\hline $18-19$ & 401 & 24.3 \\
\hline Gender & & \\
\hline Male & 883 & 53.5 \\
\hline Female & 767 & 46.5 \\
\hline Race & & \\
\hline Non-Hispanic White & 485 & 29.4 \\
\hline Non-Hispanic Black & 408 & 24.7 \\
\hline Mexican American & 376 & 22.8 \\
\hline Other Hispanic & 190 & 11.5 \\
\hline Other/Mixed & 191 & 11.6 \\
\hline Education & & \\
\hline Less than high school & 1393 & 84.4 \\
\hline High School graduate or GED & 131 & 7.9 \\
\hline More than High & 126 & 7.6 \\
\hline Ratio of family income to poverty & & \\
\hline$\leq 1$ & 540 & 32.7 \\
\hline
\end{tabular}

\subsection{Levels of urinary phthalate metabolites and liver function indicators}

Descriptive statistics for phthalate metabolites and liver function indicators are presented in Table 2. The detection rates for the eleven phthalate metabolites ranged from $71.90-99.90 \%$. The median concentrations of MECPP, MEHHP, MEHP, MEOHP, MCNP, MCOP, MnBP, MCPP, MEP, MiBP and MBzP were 7.34, 6.82, 5.03, 6.41, 5.42, 7.09, 7.29, 5.39, 8.47, 6.84 and $6.59 \mu \mathrm{g} / \mathrm{mmol} \mathrm{Cr}$ respectively. Spearman correlation analysis showed that except for MCOP and MEP, all of them were significantly correlated. Details are shown in Table S2. 
Table 2

Distribution of urinary phthalate metabolites and indicators of liver function for adolescents aged 12-19 years old in NHANES 2007-2016 $(\mathrm{N}=1,650)$

\begin{tabular}{|lllll|}
\hline & ZLOD\% & P25 & P50 & P75 \\
\hline Urinary phthalate metabolites $(\mu \mathrm{g} / \mathrm{mmol} \mathrm{Cr})$ & & & & \\
\hline MECPP & 99.90 & 6.77 & 7.34 & 7.99 \\
\hline MEHHP & 99.60 & 6.26 & 6.82 & 7.50 \\
\hline MEHP & 71.90 & 4.39 & 5.03 & 5.73 \\
\hline MEOHP & 99.70 & 5.82 & 6.41 & 7.03 \\
\hline MCNP & 98.00 & 4.95 & 5.42 & 6.01 \\
\hline MCOP & 99.60 & 6.27 & 7.09 & 8.04 \\
\hline MnBP & 98.80 & 6.74 & 7.29 & 7.79 \\
\hline MCPP & 93.10 & 4.74 & 5.39 & 6.06 \\
\hline MEP & 99.90 & 7.66 & 8.47 & 9.35 \\
\hline MiBP & 99.40 & 6.33 & 6.84 & 7.31 \\
\hline MBzP & 99.00 & 5.98 & 6.59 & 7.27 \\
\hline Liver function & & & & \\
\hline ALT (IU/L) & 100.00 & 4.30 & 4.50 & 4.70 \\
\hline AST (IU/L) & 100.00 & 7.00 & 7.20 & 7.50 \\
\hline GGT(U/L) & 100.00 & 1.09 & 1.33 & 1.57 \\
\hline ALP (IU/L) & 100.00 & 19.00 & 22.00 & 26.00 \\
\hline TBIL (mg/dL) & 100.00 & 11.00 & 13.00 & 18.00 \\
\hline ALB (g/dL) & 100.00 & 69.00 & 96.00 & 170.00 \\
\hline TP(g/dL) & 100.00 & 0.50 & 0.60 & 0.80 \\
\hline AST/ALT & 1000 & 21.00 \\
\hline M & & & & \\
\hline
\end{tabular}

\subsection{Survey-weighted liner regression analyses}

The results of survey-weighted linear regression are shown in Table S3. For every 1 unit increase in urinary In-transformed concentration of MCOP, the In-transformed concentration of TBIL decreased by 0.05 unit ( $P$ FDR $=0.001)$. There was also a negative correlation between MiBP and TBIL $(\beta=-0.06 ; P$ FDR $=0.003)$. A positive association was found between MCPP and TBIL $(\beta=0.064 ;$ P FDR $=0.011)$. $\Sigma D E H P$ $(\beta=-0.01 ; \mathrm{PFDR}=0.001), \operatorname{MCOP}(\beta=-0.009 ; \mathrm{P} F D R=0.001)$ and $\operatorname{MEP}(\beta=-0.004 ; \mathrm{PFDR}=0.018)$ were 
negatively correlated with ALB, while MCPP was positively correlated with ALB $(\beta=0.008$; P FDR $=0.005)$. Significant negative association was found for MCOP with TP $(\beta=-0.008 ; P$ FDR $=0.001)$. Nonsignificant linear relationships were found between ALT, AST, GGT, ALP, and ALT/AST with phthalate metabolites.

\subsection{BKMR analyses}

To account for the multicollinearity in the regression analysis, we used the BKMR model for further analysis. The cumulative effect of exposure to phthalate metabolite mixtures on indicators of liver function were shown in Figure 1. We observed that phthalate metabolite mixtures had significant positive dose response relationships with ALT, AST, GGT, ALP and TBIL, and significant negative dose response relationships with ALB and TP. We did not find statistically significant association between phthalate metabolite mixtures and AST/ALT. In BKMR analysis, as compared to when all of the exposures were fixed at their median value, ALT, AST, GGT, ALP and TBIL increased by $0.0075,0.0122,0.0045,0.021$ and 0.027 units, respectively, when all of the exposures were at their 75th percentile (Figure 1A,1B,1C,1D,1E). ALB and TP decreased by 0.073 and 0.072 units, respectively, when all exposures were in the 75 th percentile, compared with when all exposures were fixed at 50\% (Figure 1F, 1G). The PIP of $\sum$ DEHP was extremely high at 1 in the overall effect of phthalate mixtures on ALB. The PIP of MCOP, MnBP, MCPP, MEP, and MiBP group were exceeded the 0.5 group-PIP threshold in the overall effect of phthalate mixtures on TBIL. The PIP of MCPP group exceeded the 0.5 group-PIP threshold in the overall effect of phthalate mixtures on TB (Table S5). We further plotted the bivariate exposure response relationship of

one phthalate metabolite at different quantiles of another phthalate metabolite, and the results suggested that there were possible interactions between phthalate metabolites on liver function indicators (Figure S2- Figure S9).

\section{Discussion}

The purpose of this study was to examine the associations between urine phthalate levels and changes in liver function indicators within a representative sample of US adolescents. In the weighted linear regression models, we found associations between several phthalate metabolites and TBIL, ALB, and TP. In the BKMR analysis, our results shown that phthalate metabolite mixtures had significant positive dose response relationships with ALT, AST, GGT, ALP and TBIL, and significant negative dose response relationships with ALB and TP.

ALT was mainly distributed in liver. AST was mainly distributed in myocardium, followed by liver. Serum ALT can be sharply increased before the onset of clinical symptoms in patients with acute liver injury, while AST is significantly increased in cases of chronic hepatitis, cirrhosis, and liver cancer [14]. ALP and GGT are also abundant in liver cells. Serum ALP and GGT are significantly increased when cholestasis caused by cirrhosis, cholelithiasis and tumor (Gazzin et al.2016). Linling Yu (Yu et al.2021) reported that ¿DEHP was positively correlated with ALT, GGT, and ALP, and MBP was positively correlated with AST. Wenxin Wang (Wang et al.2015) reported that ALT, AST, GGT were significantly raised as compared to the controls with increasing plasma DEHP residues. Our study found phthalate exposure was not significantly associated with ALT, AST, GGT, and ALP, but our BKMR analysis observed that phthalate 
metabolite mixtures had significant positive overall on ALT, AST, GGT, and ALP. These differences possibly because our study only included participants aged from 12 to 19 years old, and the other two studies were based on adults. Previous studies on animal reported that the liver toxicity of phthalates was related to dose and time-dependent (. Further studies are needed to replicate these findings.

Bilirubin usually increases with excess bilirubin production (Praveena et al. 2018) (such as hemolysis), hepatocyte injury (such as hepatitis, cirrhosis, and fatty liver), or obstructed bile drainage (such as bile duct stones, pancreatic cancer, and bile duct cancer (Newsome et al. 2018). Our study found that phthalate metabolite mixtures had significant positive overall effect on TBIL. This finding is in line with previous studies that reported phthalate exposure is associated with cholestasis (Gaitantzi et al.2018; von et al. 2009). However, our study also found that MCOP and MiBP were negatively correlated with TBIL. This negative correlation may be related to the fact that phthalates are thought to be involved in inducing oxidative stress and inflammation, while TBIL is thought to have potent antioxidant properties (Ferguson et al.2012).

Hepatocytes are the main site of protein synthesis. The decrease of serum albumin and total protein levels indicates the gradual decrease of normal hepatocytes and the poor function of hepatocyte protein synthesis (Whitfield et al.2001). Our study found that LDEHP, MCOP, and MEP were negatively correlated with ALB, as well as MCOP and TP, consistent with the effect of phthalate mixtures on ALB and TP in BKMR model. This finding is consistent with previous studies that showed exposure to phthalates can lead to hepatocyte apoptosis and accelerate liver damage (Rusyn et al. 2006; Ha et al.2016; Lee et al. 2020). Our results also showed that MCPP was positively correlated with ALB. We lack the detailed knowledge to explain this positive correlation, additional studies will be required to clarify the mechanistic link between phthalate exposure and ALB.

The main strengths of this study are that we included a representative sample of US adolescents and we used the data that had been consolidated for ten years. To our knowledge, this is the first study that summarized the urine phthalate levels and seven liver function indicators in adolescents. Further, we applied the BKMR analysis to examine the joint effects of phthalate mixtures on liver function indicators. The study provides more evidence for further studies to demonstrate a correlation between phthalate exposures with liver dysfunction.

Our study has several limitations. First, the NHANES data were cross-sectional, which did not allow us to make causal inferences. Therefore, all relationships are related and further prospective research should be done to overcome this methodological limitation. Regardless, this study provides important information regarding how phthalate levels change in association with subclinical changes in liver function indicators in the U.S. adolescents which have not been previously reported. Second, because we had no information about the subjects' alcohol consumption and smoking, we did not control for these underlying variables and only adjusted for covariates such as age, BMI and sex. Finally, we measured phthalate exposure using a single-spot urine sample from each subject, possibly without taking into 
account changes in the human body over time. This may prevent us from obtaining a more precise exposure assessment to reduce exposure misclassification.

\section{Conclusions}

Phthalate metabolites were associated with changes in liver function indicators among U.S. adolescents. Further understanding about this association would be helpful for elucidating the pathogenesis of liver disorders.

\section{Declarations}

Ethics approval and consent to participate

The NHANES study was approved by the NCHS Research Ethics Review Board (Continuation of Protocol \#2011-17 http://www.cdc.gov/nchs/nhanes/irba98.htm), and all methods were performed in accordance with the relevant guidelines and regulations. All participants and parents or legal guardians of minors provided written informed consent.

Consent to Publish

All authors approved the final manuscript for submission and gave consent for publication.

\section{Author Contributions:}

STX contributed to designing this article, performing the statistical analyses, and drafting the manuscript. $\mathrm{CL}$ provided the statistical analyses. $\mathrm{XL}$ provided critical revision of the manuscript. All authors read and gave final approval of the version to be published.

\section{Funding}

This research was funded by the Hunan Provincial Natural Science Foundation youth Foundation (2021JJ40275).

\section{Conflicts of Interest:}

The authors declare no conflict of interest. The funders had no role in the design of the study; in the collection, analyses, or interpretation of data; in the writing of the manuscript, or in the decision to publish the results.

\section{Availability of data and materials}

The datasets generated during the current study are available in the CDC official website.

\section{Appendix A: Supplementary material}




\section{References}

Asrani SK, Devarbhavi H, Eaton J, Kamath PS. Burden of liver diseases in the world. J Hepatol. 2019; 70(1): 151-171. doi: 10.1016/j.jhep.2018.09.014.

Attanasio R. Sex differences in the association between perfluoroalkyl acids and liver function in US adolescents: Analyses of NHANES 2013-2016. Environ Pollut. 2019; 254(Pt B): 113061. doi: 10.1016/j.envpol.2019.113061.

Benjamin S, Masai E, Kamimura N, Takahashi K, Anderson RC, Faisal PA. Phthalates impact human health: Epidemiological evidences and plausible mechanism of action. J Hazard Mater. 2017; 340: 360383. doi: 10.1016/j.jhazmat.2017.06.036.

Bobb JF, Valeri L, Claus Henn B, Christiani DC, Wright RO, Mazumdar M, Godleski JJ, Coull BA. Bayesian kernel machine regression for estimating the health effects of multi-pollutant mixtures. Biostatistics. 2015; 16(3): 493-508. doi: 10.1093/biostatistics/kxu058.

CDC, NHANES 2015-2016 Laboratory Methods.

2016. https://wwwn.cdc.gov/nchs/nhanes/continuousnhanes/labmethods.aspx?BeginYear=2015

Ferguson KK, Loch-Caruso R, Meeker JD. Exploration of oxidative stress and inflammatory markers in relation to urinary phthalate metabolites: NHANES 1999-2006. Environ Sci Technol. 2012; 46(1): 477-85. doi: 10.1021/es202340b.

Frediani JK, Naioti EA, Vos MB, Figueroa J, Marsit CJ, Welsh JA. Arsenic exposure and risk of nonalcoholic fatty liver disease (NAFLD) among U.S. adolescents and adults: an association modified by race/ethnicity, NHANES 2005-2014. Environ Health. 2018; 17(1): 6. doi: 10.1186/s12940-017-0350-1.

Gaitantzi H, Hakenberg P, Theobald J, Heinlein H, Cai C, Loff S, Wölfl S, Ebert MP, Breitkopf-Heinlein K, Subotic U. Di (2-Ethylhexyl) Phthalate and Its Role in Developing Cholestasis: An In Vitro Study on Different Liver Cell Types. J Pediatr Gastroenterol Nutr. 2018; 66(2): e28-e35. doi:

10.1097/MPG.0000000000001813.

Gazzin S, Vitek L, Watchko J, et al. A novel perspective on the biology of bilirubin in health and disease. Trends Mol Med. 2016; 22: 758-68. doi:10.1016/j.molmed.2016.07.004

Ha M, Wei L, Guan X, Li L, Liu C. p53-dependent apoptosis contributes to di-(2-ethylhexyl) phthalateinduced hepatotoxicity. Environ Pollut. 2016; 208(Pt B): 416-25. doi: 10.1016/j.envpol.2015.10.009.

Ito Y, Kamijima M, Nakajima T. Di(2-ethylhexyl) phthalate-induced toxicity and peroxisome proliferatoractivated receptor alpha: a review. Environ Health Prev Med. 2019; 24(1): 47. doi: 10.1186/s12199-0190802-z. 
Jiao Y, Tao Y, Yang Y, Diogene T, Yu H, He Z, Han W, Chen Z, Wu P, Zhang Y. Monobutyl phthalate (MBP) can dysregulate the antioxidant system and induce apoptosis of zebrafish liver. Environ Pollut. $2020,257,113517$.

Katsikantami I, Sifakis S, Tzatzarakis MN, Vakonaki E, Kalantzi OI, Tsatsakis AM, Rizos AK. A global assessment of phthalates burden and related links to health effects. Environ Int. 2016; 97: 212-236. doi: 10.1016/j.envint.2016.09.013.

Lawlor DA, Callaway M, Macdonald-Wallis C, Anderson E, Fraser A, Howe LD, Day C, Sattar N. Nonalcoholic fatty liver disease, liver fibrosis, and cardiometabolic risk factors in adolescence: a crosssectional study of 1874 general population adolescents. J Clin Endocrinol Metab. 2014; 99(3): E410-7. doi: 10.1210/jc.2013-3612.

Lee CY, Suk FM, Twu YC, Liao YJ. Long-Term Exposure to Low-Dose Di-(2-ethylhexyl) Phthalate Impairs Cholesterol Metabolism in Hepatic Stellate Cells and Exacerbates Liver Librosis. Int J Environ Res Public Health. 2020; 17(11): 3802. doi: 10.3390/ijerph17113802.

Malin AJ, Lesseur C, Busgang SA, Curtin P, Wright RO, Sanders AP. Fluoride exposure and kidney and liver function among adolescents in the United States: NHANES, 2013-2016. Environ Int. 2019; 132: 105012. doi: 10.1016/j.envint.2019.105012.

Newsome PN, Cramb R, Davison SM, Dillon JF, Foulerton M, Godfrey EM, Hall R, Harrower U, Hudson M, Langford A, Mackie A, Mitchell-Thain R, Sennett K, Sheron NC, Verne J, Walmsley M, Yeoman A. Guidelines on the management of abnormal liver blood tests. Gut. 2018; 67(1): 6-19. doi: 10.1136/gutjnl2017-314924.

Odebeatu CC, Taylor T, Fleming LE, J Osborne N. Phthalates and asthma in children and adults: US NHANES 2007-2012. Environ Sci Pollut Res Int. 2019; 26(27): 28256-28269. doi: 10.1007/s11356-01906003-2.

Praveena SM, Teh SW, Rajendran RK, Kannan N, Lin CC, Abdullah R, Kumar S. Recent updates on phthalate exposure and human health: a special focus on liver toxicity and stem cell regeneration. Environ Sci Pollut Res Int. 2018; 25(12):11333-11342. doi: 10.1007/s11356-018-1652-8.

Rusyn I, Peters JM, Cunningham ML. Modes of action and species-specific effects of di-(2ethylhexyl)phthalate in the liver. Crit Rev Toxicol. 2006 ;36(5): 459-79. doi: $10.1080 / 10408440600779065$.

Shah-Kulkarni S, Lee S, Jeong KS, Hong YC, Park H, Ha M, Kim Y, Ha EH. Prenatal exposure to mixtures of heavy metals and neurodevelopment in infants at 6 months. Environ Res. 2020 ; 182: 109122. doi: 10.1016/j.envres.2020.109122. 
von Rettberg H, Hannman T, Subotic U, Brade J, Schaible T, Waag KL, Loff S. Use of di(2ethylhexyl)phthalate-containing infusion systems increases the risk for cholestasis. Pediatrics. 2009; 124(2): 710-6. doi: 10.1542/peds.2008-1765.

Wang W, Leung AOW, Chu LH, Wong MH. Phthalates contamination in China: Status, trends and human exposure-with an emphasis on oral intake. Environ Pollut. 2018; 238: 771-782. doi:

10.1016/j.envpol.2018.02.088.

Whitehead MW, Hawkes ND, Hainsworth I, et al. A prospective study of the causes of notably raised aspartate aminotransferase of liver origin. Gut 1999; 45: 129-33. doi:10.1136/gut.45.1.129

Whitfield JB. Gamma glutamyl transferase. Crit Rev Clin Lab Sci. 2001; 38(4): 263-355. doi: $10.1080 / 20014091084227$.

Wang W, Xu X, Fan CQ. Health hazard assessment of occupationally di-(2-ethylhexyl)-phthalate-exposed workers in China. Chemosphere. 2015; 120: 37-44. doi: 10.1016/j.chemosphere.2014.05.053.

Wolff MS, Teitelbaum SL, Pinney SM, Windham G, Liao L, Biro F, Kushi LH, Erdmann C, Hiatt RA, Rybak ME, Calafat AM; Breast Cancer and Environment Research Centers. Investigation of relationships between urinary biomarkers of phytoestrogens, phthalates, and phenols and pubertal stages in girls. Environ Health Perspect. 2010; 118(7): 1039-46. doi: 10.1289/ehp.0901690.

Yu L, Yang M, Cheng M, Fan L, Wang X, Xu T, Wang B, Chen W. Associations between urinary phthalate metabolite concentrations and markers of liver injury in the US adult population. Environ Int. 2021; 155: 106608. doi: 10.1016/j.envint.2021.106608.

Zhang Q, Zhao Y, Talukder M, Han Y, Zhang C, Li XN, Li JL. Di(2-ethylhexyl) phthalate induced hepatotoxicity in quail (Coturnix japonica) via modulating the mitochondrial unfolded protein response and NRF2 mediated antioxidant defense. Sci Total Environ. 2019; 651(Pt 1): 885-894. doi: 10.1016/j.scitotenv.2018.09.211.

Zipf G, Chiappa M, Porter KS, Ostchega Y, Lewis BG, Dostal J. National health and nutrition examination survey: plan and operations, 1999-2010. Vital Health Stat 1. 2013; (56): 1-37.

\section{Figures}



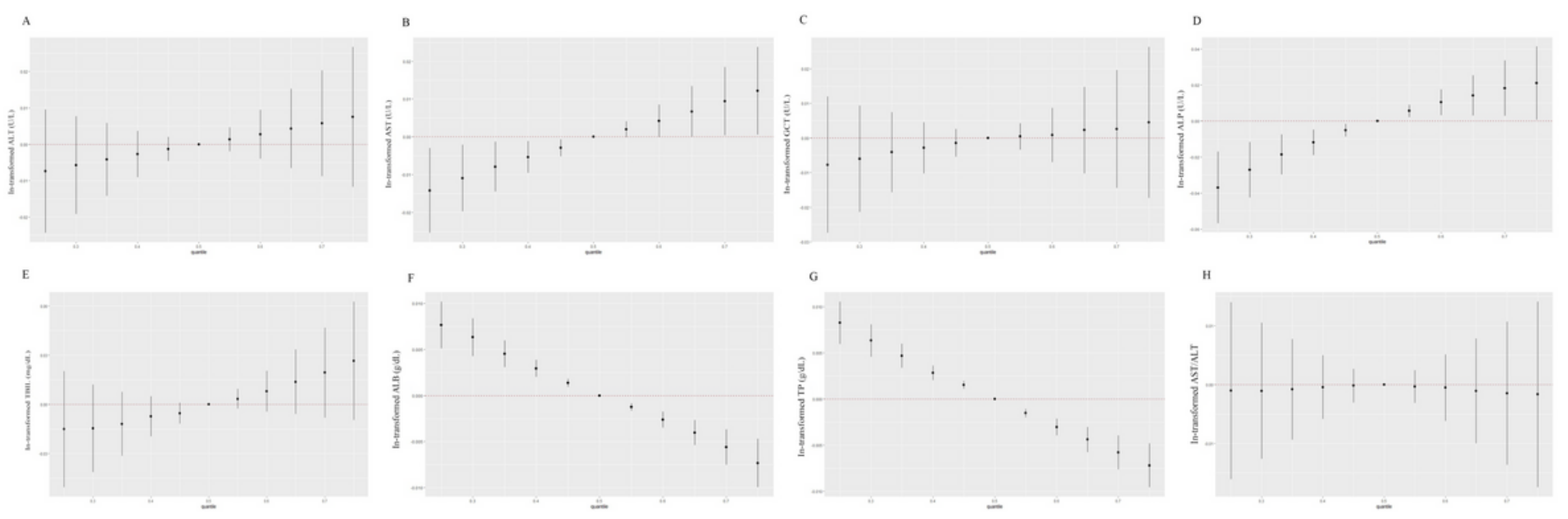

\section{Figure 1}

To account for the multicollinearity in the regression analysis, we used the BKMR model for further analysis. The cumulative effect of exposure to phthalate metabolite mixtures on indicators of liver function were shown in Figure 1.

\section{Supplementary Files}

This is a list of supplementary files associated with this preprint. Click to download.

- Supplementalfile.docx 